\title{
Procedure Planned Time Point Number
}

National Cancer Institute

\section{Source}

National Cancer Institute. Procedure Planned Time Point Number. NCI Thesaurus. Code

C117535.

The numerical identifier of a planned procedure point in time. 